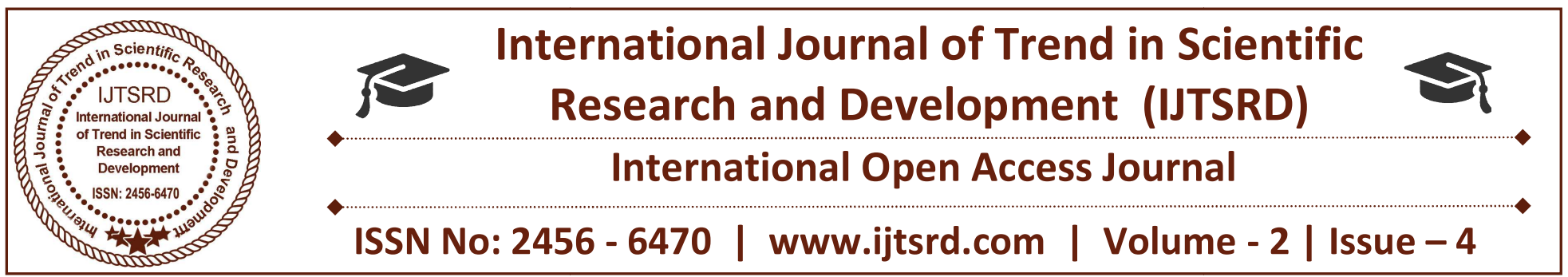

\title{
Determination of Dispersivity in A Laboratory Soil Column
}

\author{
M. R. Namitha ${ }^{1}$, V. Ravikumar ${ }^{2}$ \\ ${ }^{1}$ Post Graduate Student, ${ }^{2}$ Professor and Head \\ Department of Soil and Water Conservation and Agricultural Structures, \\ Tamil Nadu Agricultural University, Tamil Nadu, India
}

\begin{abstract}
In this study, a laboratory soil column experiment was carried out for estimating the dispersivity $(\lambda)$ in sandy loam soil by the inversion of the AdvectionDispersion Equation. An $89 \mathrm{~cm}$ deep and $15 \mathrm{~cm}$ diameter cylindrical column was filled with sandy loam soil in such a way that the bulk density of the soil in field condition was strictly satisfied in the column. Potassium bromide was used as the conservative tracer in the study. The tracer concentration was allowed to pass through the column at a constant rate and the leachate collected at different time periods were tested for the concentration of bromide. The experiment was carried out until the relative concentration $(\mathrm{c} / \mathrm{c} 0)$ has reached 1. The calculated relative concentration was plotted against time for obtaining the observed breakthrough curve (BTC). Another BTC was plotted using the analytical solution of Advection Dispersion Equation. The effective dispersion coefficient (De)value which gave the best fit between these two curves was chosen by trial and error method and the dispersivity of the soil was calculated $(\mathrm{De}=\lambda \mathrm{v})$ for a constant pore water velocity (v). The dispersivity of the sandy loam soil under study was found to be $1.7 \mathrm{~cm}$ which matches well with the previous researches.
\end{abstract}

Keywords: Dispersivity: Advection Dispersion Equation, Effective dispersion coefficient, Laboratory soil column.

\section{INTRODUCTION}

Understanding solute transport processes between the soil surface and the groundwater table is essential for limiting or effectively managing soil and groundwater pollution. Dispersivity are typically derived either from observed depth profiles of inert tracer concentrations or from breakthrough curves (BTC) of tracer concentrations that are measured in the effluent of columns or that are measured in a soil profile using solution samplers or other devices such as time domain reflectometry (TDR) probes that monitor solute concentrations.

The dispersivity $(\lambda)$ reflects the degree of mechanical mixing of solutes, caused by variations in the pore water velocity. The soil-column experiment was widely used to evaluate the transport model and determine the fate and migration of contaminants through soils. The value of $\lambda$ has traditionally been considered under saturated water condition and usually reported in the range of $0.1-2 \mathrm{~cm}$ for homogeneous saturated soils (Bear, 1972).Silliman et al., (1987) studied the scale effect in dispersion of solutes in porous media under laboratory conditions and the results for various sand-packing arrangements revealed that the breakthrough curves for uniform coarse sand showed a constant dispersivity of approximately $0.02 \mathrm{~m}$. Huang, K. et al., (1995) conducted an experimental investigation of solute transport in large, homogeneous and heterogeneous, saturated soil columns. Values of the dispersivity $(\lambda)$ for the homogeneous sandy column ranged from 0.1 to $5.0 \mathrm{~cm}$, while those for the heterogeneous column were as high as $200 \mathrm{~cm}$.

\section{MATERIALS AND METHODS \\ Laboratory Soil Column Experiment}

Tracer (Bromide) movement through the saturated agricultural soil was investigated in soil columns. The dispersivity of bromide ions in sandy loam soil was measured in this study. Potassium bromide was used 
as the conservative tracer. Bromide salts have proven to serve as excellent tracer materials since they are highly soluble, non-degradable, relatively inexpensive, and can be measured quantitatively in very low concentrations.

A cylindrical laboratory soil column of $89 \mathrm{~cm}$ height and $15 \mathrm{~cm}$ diameter (Fig. 1) was used to find the dispersion of potassium bromide tracer in a homogeneously packed sandy loam soil. The bulk density of the soil measured using core cutter method was found to be $1.58 \mathrm{~g} \mathrm{~cm}^{-3}$. The soil column was filled in such a way that the bulk density of the soil in the field was strictly satisfied in the laboratory soil column.

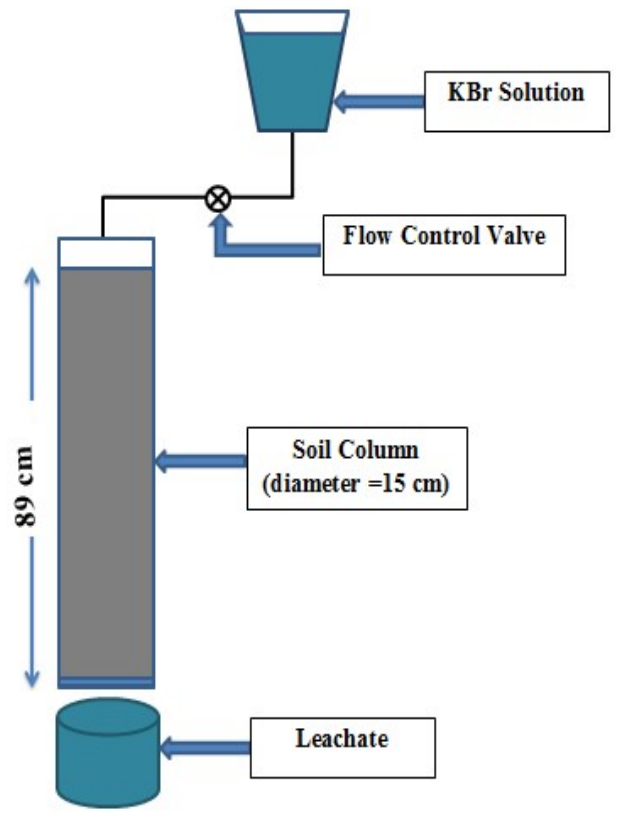

Fig. 1: Laboratory Soil Column Experimental Setup

$$
\begin{aligned}
& \text { Volume of the soil column, } \\
& \begin{array}{r}
V=\pi r^{2} h \\
\quad=\pi \times(7.5)^{2} \times(89)=15719.63 \mathrm{~cm}^{3}
\end{array} \\
& \text { Bulk Density of the soil }(B D)=\frac{M}{V}
\end{aligned}
$$

where, $M$ is the mass of the soil, $\mathrm{g}$ and, $V$ is the volume of soil, $\mathrm{cm}^{3}$.

Mass of the soil to be filled in the soil column,

$$
\begin{aligned}
M & =B D \times V \\
& =1.58 \times 15719.63 \\
& =24837.01 \mathrm{~g}=24.84 \mathrm{~kg}
\end{aligned}
$$

i.e. for satisfying the bulk density of the soil $(1.58 \mathrm{~g}$ $\left.\mathrm{cm}^{-3}\right), 24.84 \mathrm{~kg}$ of soil was filled in the soil column providing an additional free board of $1 \mathrm{~cm}$ at the top. The porosity of the soil was found to be 0.37 (Eq. 4).
Porosity $=1-\frac{\text { Bulk Density }}{\text { Particle Density }}$

The uniform soil column was saturated initially by passing water through it. The potassium bromide $(\mathrm{KBr})$ solution having an initial concentration of $20 \mathrm{~g}$ per $200 \mathrm{ml}$ of tap water was allowed to pass through the soil column from its upper end and the leachate was collected at the lower end. The input solution was given at a constant rate of $800 \mathrm{~cm}^{3} \mathrm{~h}^{-1}$ throughout the experiment. Since a constant input of solute at a given concentration $\left(c_{0}\right)$ was given at the surface boundary, this is known as step input. Concentration of bromide ions in the leachate collected at different time steps were determined by Mohr's (Argentometric titration) method.

\section{Mechanism of solute transport in soils}

'The solute conservation equation states that the change in total solute concentration $\left(C_{T}\right)$ in units of mass per volume of soil in a representative elementary volume over time is equal to the negative of the rate of change of the solute flux with depth, minus any source/ sink $(S)$ in units of mass per volume per time (Radcliff and Simunek, 2010). Only solute flow in the vertical direction ( $z$ positive up) is considered for simplification purposes.

$\frac{\partial C_{T}}{\partial t}=-\frac{\partial J_{S}}{\partial z}-S$

where, $C_{T}$ is the total solute concentration in units of mass per volume of soil, $S$ is the source/ sinks in units of mass per volume per time and, $J_{S}$ is the total solute flux.

This is the partial differential equation (PDE) with independent variables $z$ and $t$ and the dependent variable $C_{T}$. The units of each term are $\left[\mathrm{ML}^{-3} \mathrm{~T}^{-\mathrm{I}}\right]$. Solute flux $\left(J_{s}\right)$ is the rate at which the solute flows through a given cross-sectional area.

The total solute concentration $\left(C_{T}\right)$ consists of the dissolved or liquid-phase solute concentration $(c)$, expressed as a mass of solute per volume of liquid, and the adsorbed or solid-phase solute concentration $(s)$, expresses as a mass of solute per mass of soil. To convert each of these concentrations to units of mass per volume of soil, $c$ is multiplied by $\theta$ and $s$ is multiplied by $\rho_{b}$.

$C_{T}=\theta c+\rho_{b} s$

where, $c$ is theliquid-phase solute concentration $\left[\mathrm{ML}^{-}\right.$ $\left.{ }^{3}\right], s$ is the solid-phase solute concentration $\left[\mathrm{MM}^{-1}\right], \theta$ 
is the volumetric water content $\left[\mathrm{L}^{3} \mathrm{~L}^{-3}\right]$ and, $\rho_{b}$ is the bulk density of soil $\left[\mathrm{ML}^{-3}\right]$.

Solute flux in the liquid consists of three components that represent different transport processes: advective (also called convective) flux $\left(J_{I C}\right)$, diffusive flux $\left(J_{I d}\right)$ and hydrodynamic dispersive flux $\left(J_{I h}\right)$.

$J_{S}=J_{I C}+J_{I d}+J_{I h}$

\section{Advection Dispersion (Convection- Dispersion) Equation}

Most mechanistic transport models for solutes in porous materials are based on the convectiondispersion equation (CDE). The $\mathrm{CDE}$ is a partial differential equation representing mass continuity for movement of a given solute in a porous medium by dispersion and convection under specified initial and boundary conditions. Appropriate terms are incorporated into to the $\mathrm{CDE}$ to account for chemical and/or physical sink/source interactions between the solute and porous medium. The $\mathrm{CDE}$ can be developed microscopically based on Brownian motion, or macroscopically, based on Fick's law.

The final solution to the Advection-Dispersion equation obtained by the Laplace transform method is:

$$
\begin{aligned}
& c(z, t) \\
& =c_{0}\left[\sqrt{\frac{v^{2} t}{\pi D_{e}}} \exp \left(-\frac{(z-v t)^{2}}{4 D_{e} t}\right)\right. \\
& +\frac{1}{2} \operatorname{erfc}\left(\frac{z-v t}{\sqrt{4 D_{e} t}}\right) \\
& -\frac{1}{2}\left(1+\frac{v z}{D_{e}}\right. \\
& \left.\left.+\frac{v^{2} t}{D_{e}}\right) \exp \left(\frac{v z}{D_{e}}\right) \operatorname{erfc}\left(\frac{z+v t}{\sqrt{4 D_{e} t}}\right)\right]
\end{aligned}
$$

where, erfc is the complementary error function defined as $\operatorname{erfc}(x)=\frac{2}{\sqrt{\mu}} \int_{x}^{\infty} e^{-x^{2}} d x, \quad c \quad$ is the concentration at depth $z$ at time $t\left[\mathrm{ML}^{-3}\right], c_{0}$ is the initial concentration $\left[\mathrm{ML}^{-3}\right], D_{e}$ is the effective dispersion coefficient $\left[\mathrm{L}^{2} \mathrm{~T}^{-1}\right], v$ is the pore water velocity $\left[\mathrm{LT}^{-1}\right], z$ is the depth $[\mathrm{L}]$ and, $t$ is the time [T].

Effective dispersion coefficient, $D_{e}=\lambda v$ where, $\lambda$ is the dispersivity, $[\mathrm{L}]$ and, $v$ is the pore water velocity, $\left[\mathrm{LT}^{-1}\right]$.

Determination of Bromide Ion Concentration by Argentometric Titration (Mohr's method)

Mohr's method determines the bromide ion concentration of a solution by titration with silver nitrate $\left(\mathrm{AgNO}_{3}\right)$. As the silver nitrate solution is slowly added, a precipitate of silver bromide forms.

$\mathrm{Ag}_{(\mathrm{aq})}^{+}+\mathrm{Br}_{(\mathrm{aq})}^{-} \rightarrow \mathrm{AgBr}_{(\mathrm{s})}$

The end point of the titration occurs when all the bromide ions were precipitated. Then additional silver ions react with the chromate ions of the indicator, potassium chromate, to form a red-brown precipitate of silver chromate.

$$
2 \mathrm{Ag}^{+}{ }_{(\mathrm{aq})}+\mathrm{CrO}^{2-}{ }_{(\mathrm{aq})} \rightarrow \mathrm{Ag}_{2} \mathrm{CrO}_{4(\mathrm{~s})}
$$

The $\mathrm{pH}$ of the sample solutions should be between 7 and 10 because chromate ion is the conjugate base of the weak chromic acid. A suitable $\mathrm{pH}$ was achieved by saturating the analyte solution with sodium hydrogen carbonate $\left(\mathrm{NaHCO}_{3}\right)$.

Although the silver bromide that forms is a white precipitate, the chromate indicator initially gives the cloudy solution a faint lemon-yellow colour. The endpoint of the titration was identified as the first appearance of a red-brown colour of silver chromate. The titration was repeated with further aliquots of diluted samples until concordant results were obtained.

A blank titration is carried out by titrating a fixed and known concentration of titrant into a solvent with zero analyte. The only difference from the regular titration is the absence of analyte. This allows the amount of reactive substance within the plain solvent to be determined and hence allows a determination of the error in future titration experiments using this solvent.The blank titration was conducted to correct the errors that might occur in the titre values due to the presence of chloride impurities in the potassium chromate indicator. The volume of $\mathrm{AgNO}_{3}$ needed for the complete precipitation of the bromide ions (titre value) was noted and the concentration of bromide ions in the leachate was calculated using the following equation:

$$
C=\frac{\left(V_{S}-V_{B}\right) \times M_{A g N O 3} \times \text { Equivalent wt of } \mathrm{Br} \times 1000}{\text { Vol.of sample taken }}
$$


where, $c$ is the concentration of bromide in the leachate in $\mathrm{mg} \mathrm{l}^{-1}, V_{S}$ is the volume of $\mathrm{AgNO}_{3}$ needed for the sample in $\mathrm{ml}, V_{B}$ is the volume of $\mathrm{AgNO}_{3}$ needed for the blank in $\mathrm{ml}(0.2 \mathrm{ml})$ and, $\mathrm{M}_{\mathrm{AgNO} 3}$ is themolarity of $\mathrm{AgNO}_{3}(0.1 \mathrm{M})$. The equivalent weight of bromide is $79.9 \mathrm{~g}$.

The concentration $(c)$ of the leachate and the relative concentrations $\left(c / c_{0}\right)$ for different time steps were calculated using Eq. 10. Once the concentration of the leachate was calculated, a graph was plotted with the relative $\left(c / c_{0}\right)$ against the time $(t)$. This graph is said to be the observed breakthrough curve.

\section{Break Through Curves (BTCs)}

The concentrations can be plotted as a function of time at a given depth for various values of effective dispersion coefficient $\left(D_{e}\right)$ and the plots are called breakthrough curves (BTC). Break through curves are extensively used to characterize the physicochemical processes involved in the transport of solutes in porous media. Analysis of column BTCs can provide useful estimates of the parameters for the physicochemical processes involved in subsurface solute transport (Radcliff and Simunek, 2010). Such parameters are essential for developing and validating theoretical models for solute transport.

The relative concentration $\left(c / c_{0}\right)$ obtained from the Advection-Dispersion Equation (Eq. 8) was plotted against corresponding time periods $(t)$ to generate the BTC (predicted) at $89 \mathrm{~cm}$ depth. Microsoft excel was effectively utilised to calculate the relative concentration values. Excel allows the user to calculate the complementary error function directly. The effective dispersion coefficient is a constant, rather than a function of time or mean travel distance (Dagan, 1988; Neuman and Zhang, 1990; Rajaram and Gelhar, 1993). The effective dispersion coefficient $\left(D_{e}\right)$ in Eq. (8) was chosen arbitrarily by trial and error method.

The pore water velocity $(v)$ was calculated as:

$v=\frac{J_{W}}{\theta}$

where, $\theta$ is the porosity and, $J_{W}$ is the Darcy velocity.

$J_{W}=\frac{Q}{A}$

where, $Q$ is the constant discharge rate of the step input $\left(800 \mathrm{~cm}^{3} \mathrm{~h}^{-1}\right)$ and $A$ is the input surface area of the soil column in $\mathrm{cm}^{2}$. The pore water velocity $(v)$ was calculated as $12.24 \mathrm{~cm} \mathrm{~h}^{-1}$ (i.e. $293.79 \mathrm{~cm} \mathrm{~d}^{-1}$ ).

The BTC for observed bromide concentration was obtained using Eq. 10 and the resulting plot was fitted to the solution of 1-D ADE (Eq. 8). The effective dispersion coefficient $\left(D_{e}\right)$ which gives the best fit between the observed and predicted BTC's was chosen by trial and error method. Finally, the dispersivity $(\lambda)$ for the bromide ions in the sandy loam soil column at a depth of $89 \mathrm{~cm}$ was calculated using Eq. 9.

\section{RESULTS AND DISCUSSIONS}

The hydrodynamic dispersion coefficient $\left(D_{e}\right)$ and the pore water velocity $(v)$ were derived from depth profile $(89 \mathrm{~cm})$ assuming that $v$ and $D_{e}$ are constant in the soil profile and do not change with depth and time, i.e. a hydro dynamically homogeneous soil profile and steady state flow conditions. The analytical solutions of the solute transport equation (ADE) was derived and fitted to the observed BTCs.

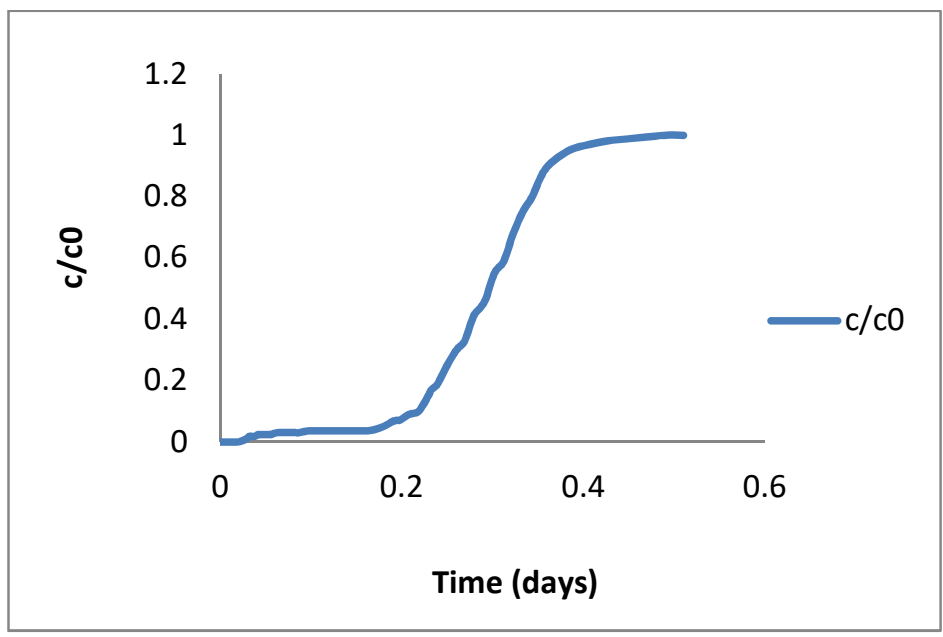

\section{Fig. 2: Relative Concentration vs. Time curve obtained from the estimated bromide concentration}

An initial concentration of $20 \mathrm{~g}$ per $200 \mathrm{ml}$ of potassium bromide was given as the input tracer solution. Initially, the concentration of bromide was 0 in the leachate and as time passes the bromide starts to appear in the leachate. The first appearance of bromide was found 43 minutes after the starting of the experiment. The concentration of bromide in the leachate increased with time and reached $100 \%$ concentration after $12.25 \mathrm{hrs}$.

The bromide concentration of the leachate (c) estimated using Mohr's test was determined by Eq. (10) and the relative concentration $\left(c / c_{0}\right)$ for different 
time steps were calculated. The observed BTC (Fig. 2 ) in the homogeneous sandy loam column was found to be a smooth and sigmoidal (S-shaped) curve. After about $6.67 \mathrm{hrs}$, the relative concentration reached a value of 0.5 and half of the BTC had arrived. Eventually, concentration reached the input concentration at $12.25 \mathrm{hrs}$. At this point, solute has arrived at this depth even in the smallest pores.

The effective dispersion coefficient $\left(D_{e}\right)$ and dispersivity $(\lambda)$ were determined by fitting the analytical solution of the convection-dispersion equation (Eq. 8) to the BTC obtained by estimated relative concentration. The solution of 1-D ADE was fitted to the locally measured BTC by randomly changing the value of effective dispersion coefficient $\left(D_{e}\right)$ by trial and error method. Since the dispersion of the solute front was affected by $\mathrm{D}_{\mathrm{e}}$, so was the BTC. As $\mathrm{D}_{\mathrm{e}}$ increased, the BTC became more dispersed.

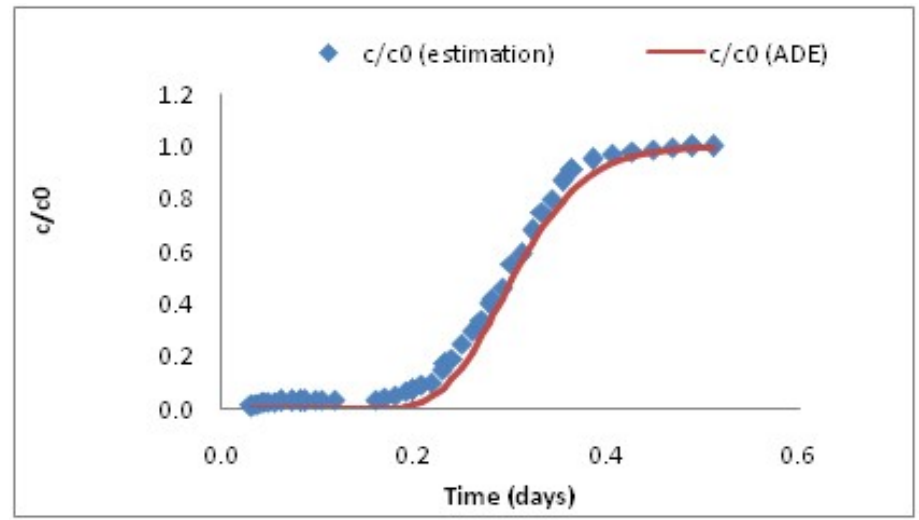

Fig. 3: Observed and Fitted BTC using ADE

Fig. 3 shows the curves fitted using Microsoft Excel. The average pore water velocity was assumed to be a constant of $293.79 \mathrm{~cm} \mathrm{~d}^{-1}$ throughout the soil profile. The best fit between the two curves were obtained when $D_{e}$ value was set to $500 \mathrm{~cm}^{2} \mathrm{~d}^{-1}$. The longitudinal dispersivity $(\lambda)$ was calculated using Eq. 9. The value of dispersivity $(\lambda)$ for bromide at the depth of $89 \mathrm{~cm}$ for the proposed sandy loam was calculated as $1.70 \mathrm{~cm}$. This value lies well within the range $(0.1-2 \mathrm{~cm})$ reported for homogeneous saturated soils in laboratory column experiments (Bear, 1972).

\section{Summary and Conclusion}

The determination of various solute transport parameters is important mainly in the groundwater pollution studies. In present study, the dispersivity of bromide ions in a laboratory soil column was determined by the inversion of Advection-Dispersion Equation. The dispersivity of the bromide ions in a homogeneous sandy loam soil column was found to be $1.7 \mathrm{~cm}$.

\section{REFERENCES}

1. Bear, J. 1972. Dynamics of Fluids in Porous Media. American Elsevier Pub. Co.

2. Radcliff, D. E., and J. Simunek. 2010. Soil Physics with Hydrus: Modeling and Applications. CRC Press: 183-347.

3. Dagan, G. 1988. Theory of Solute Transport by Groundwater. Annual Reviews of Fluid Mechanics 19: 183-215.

4. Neuman, S. P., and Y. K., Zhang. 1990. A QuasiLinear Theory of Non-Fickian and Fickian Subsurface Dispersion: 1. Theoretical Analysis with Application to Isotropic Media. Water Resour. Res. 26(5): 887-902.

5. Rajaram, H., and L. W. Gelhar. 1993. Plume-scale dependent dispersion in heterogeneous aquifers: 2 . Eulerian analysis and three-dimensional aquifers. Water Resour. Res. 29(9): 3261-3276.

6. Silliman, S. E. and, E. S. Simpson. 1987. Laboratory evidence of the scale effect in dispersion of solutes in porous media. Water Resour. Res. 23(8): 1667-1673.

7. Huang, K., N. Toride, and M. Th. van Genuchten. 1995. Experimental investigation of solute transport in large, homogeneous and heterogeneous, saturated soil columns. Transport in Porous Media 18: 283-302. 